\title{
ON BAIRE-HYPERPLANE SPACES
}

\author{
by M. VALDIVIA \\ (Received 28th April 1978)
}

In this article we prove that in every infinite dimensional separable Fréchet space there is a dense barrelled subspace which is not the inductive limit of Bairehyperplane spaces.

The linear spaces we use are defined over the field $K$ of the real or complex numbers. By "space" we mean "separated locally convex topological vector space". Given a space $H, H^{\prime}$ denotes its topological dual and if $A$ is a bounded closed absolutely convex subset of $H, H_{A}$ is the normed space over the linear hull of $A$ has as norm the gauge on $A$. Let $\mathscr{A}$ be the family of all the bounded closed absolutely convex subsets of $H$. If $B$ is a subspace of $H$ its local closure $\tilde{B}$ is the intersection of all subspaces of $H$ containing $B$ and intersecting $H_{A}, A \in \mathscr{A}$, in a closed set. We say that $H$ is locally complete if $H_{A}$ is a Banach space for every $A \in \mathscr{A}$.

Let $\Omega$ be a non void open set in the euclidean $m$-dimensional space $R^{m}$. We denote by $\mathscr{D}(\Omega)$ and $\mathscr{D}^{\prime}(\Omega)$ the spaces of $L$. Schwartz with the strong topologies. Let $M$ be the set of all points of $\Omega$ having rational coordinates. If $b \in M, T_{b}$ is the set formed by the delta of Dirac concentrated in $b$ and its derivatives of all orders. $\mathscr{D}_{0}^{\prime}(\Omega)$ is the subspace of $\mathscr{D}^{\prime}(\Omega)$ generated by $\left\{T_{b}: b \in M\right\}$. As is usual, $C \sim D$ is the difference of $C$ and $D$. $N$ denotes the set of the natural numbers.

\section{Baire-hyperplane spaces}

A space $H$ is a Baire-hyperplane space if every union of a countable family of closed hyperplane of $\boldsymbol{H}$ has void interior.

The following three theorems are of a trivial nature.

Theorem 1. Every separated quotient of a Baire-hyperplane space is a Bairehyperplane space.

Theorem 2. If a space $E$ contains a dense subspace which is a Baire-hyperplane space then $H$ is a Baire-hyperplane space.

Theorem.3. If $E$ is a Baire-hyperplane space and $\mathscr{T}$ is a separated locally convex topology on $E$ coarser than the original topology, then $E[\mathscr{T}]$ is a Baire-hyperplane space.

Note 1. If $E$ is a space such that its topology is defined by a family of norms there is in $E^{\prime}\left[\sigma\left(E^{\prime}, E\right)\right]$ a compact absolutely convex subset $A$ which is total. Then, if $\mathscr{F}$ is a 
topology on $E^{\prime}$ compatible with the dual pair $\left\langle E^{\prime}, E\right\rangle$, Theorem 3 implies that $E_{A}^{\prime}$ with the topology induced by $\mathscr{F}$ is a Baire-hyperplane space. According to Theorem 2, $E^{\prime}[\mathscr{F}]$ is a Baire-hyperplane space.

Theorem 4. Every metrizable (LF)-space is a Baire-hyperplane space.

Proof. Let $\left(H_{n}\right)$ be an increasing sequence of subspaces of $E$ with $\cup\left\{H_{n}\right.$ : $n \in N\}=E$. Suppose that there is a topology on $H_{n}, \mathscr{F}_{n}$, finer than the original topology such that $H_{n}\left[\mathscr{F}_{n}\right], n \in N$, is a Fréchet space and $E$ is the inductive limit of the sequence $\left(H_{n}\left[\mathscr{F}_{n}\right]\right)$. The sequence of the closures of $H_{n}$ in $E, n \in \mathrm{N}$, is increasing and its union in $E$. Since $E$ is a metrizable barrelled space there is a positive integer $p$ so that $\bar{H}_{p}=E$, (1). On the other hand, let us suppose that there is a sequence $\left(L_{n}\right)$ of closed hyperplanes of $E$ with $E$ as its union. Then $\cup\left\{L_{n} \cap H_{p}\left[\mathscr{F}_{p}\right]: n \in N\right\}=H_{p}\left[\mathscr{T}_{p}\right]$ and since $H_{p}\left[\mathscr{T}_{p}\right]$ is a Fréchet space there is a positive integer $q$ such that $L_{q} \supset$ $H p\left[\mathscr{T}_{p}\right]$ and therefore $L_{q} \supset \bar{H}_{p}=E$ which is a contradiction.

Note 2. In (3, p. 369) a non closed barrelled subspace of $l^{1}$ is given. It is easy to show it is not a Baire-hyperplane space. Other examples of normed barrelled spaces which are not Baire-hyperplane spaces are given in (2).

Theorem 5. Every product of Baire-hyperplane spaces is a Baire-hyperplane space.

Proof. Let $\left\{E_{i} ; i \in I\right\}$ be a family of Baire-hyperplane spaces and let $E$ be its topological product. If we suppose that $E$ is not a Baire-hyperplane space let $\left(L_{n}\right)$ be a sequence of closed hyperplanes of $E$ covering $E$. For each $i \in I$, let $\mathscr{L}_{i}$ be the family of those elements of $\left(L_{n}\right)$ not containing $E_{i} . \mathscr{L}_{i}$ does not cover $E_{i}$ since $E_{i}$ is a Baire-hyperplane space and therefore a vector $x_{i} \in H_{i}$ can be found which is not in any member of $\mathscr{L}_{i}$. Call $F_{i}$ the linear hull of $\left\{x_{i}\right\}$. Every element of the family $\mathscr{L}=\left\{\mathscr{L}_{i} ; i \in I\right\}$ does not contain $F=\Pi\left\{F_{i}: i \in I\right\}$, but $F$ is a Baire space and thus $\mathscr{L}$ cannot cover $F$ and therefore $\mu=\left\{L_{1}, L_{2}, \ldots, L_{n}, \ldots\right\} \sim L$ is not void which means that every $M \in \mathcal{M}$ contains $E_{i}, i \in I$, and, therefore $M \supset E$ which is a contradiction.

\section{Bornological barrelled spaces}

Proposition 1. Let $H$ be a locally complete space and let $S$ and $T$ be subspaces of $E$ such that $\tilde{T} \subset S$. If $\mathscr{F}$ denotes the associated ultrabornological topology to $S$ then $T$ is $\mathscr{F}$-dense in $\tilde{T}$.

Proof. Let $T^{*}$ be the $\mathscr{F}$-closure in $S$ of $T$ and write $T_{1}$ for $T^{*} \cap \tilde{T}$. We show that $\tilde{T} \subset T^{*}$. Given any bounded closed absolutely convex subset $A$ of $H$ we have that $H_{A}$ is a Banach space and $H_{A} \cap \tilde{T}$ is a closed subspace of $H_{A}$ coinciding with $H_{A \cap \tilde{T}}$ which is therefore a Banach space and its topology is finer than the topology induced by $\mathscr{F}$ and thus $H_{A \cap \bar{T}} \cap T^{*}$, which coincides with $H_{A \cap T_{1}}$, is closed in $H_{A}$. Therefore $T_{1}$ coincides with $\tilde{T}$ and the conclusion follows. 
Next we consider a locally complete space $E$ which has a bi-orthogonal sequence $\left(x_{n}, u_{n}\right)$ such that the linear hull $G$ of $\left(x_{n}\right)$ is dense in $E$. If $x \in E$ we write

$$
\Delta(x)=\left\{n \in N: u_{n}(x) \neq 0\right\}
$$

Suppose that there is a $x_{0} \in E$ such that $\Delta\left(x_{0}\right)$ is infinite and call $\mathscr{g}$ the filter on $N$ such that $F \in \mathscr{J}$ if and only if $F \cap \Delta\left(x_{0}\right)$ has a finite complement in $\Delta\left(x_{0}\right)$ and let $\mathcal{U}$ be an ultrafilter on $\mathrm{N}$ finer than $\mathscr{J}$. For any $U \in \mathcal{U}$ we write $L(U)$ to denote the local closure of the linear hull of $\left\{x_{n}: n \in N \sim U\right\}$. Since $L\left(U_{1}\right) \cup L\left(U_{2}\right) \subset L\left(U_{1} \cap U_{2}\right)$ for every pair $U_{1}, U_{2} \in \mathscr{U}$ it follows that $L=\cup\{L(U): U \in \mathscr{U}\}$ is a subspace of $E$ containing $G$. We write $F$ for the linear hull of $L \cup\left\{x_{0}\right\}$. In what follows $\mathscr{F}$ denotes the associated ultrabornological topology to $L$.

Proposition 2. The neighbourhoods of the origin in $L[\mathscr{F}]$ absorb the bounded sets of $G$.

Proof. Let $V$ be an absolutely convex neighbourhood of the origin in $L[\mathscr{T}]$ and suppose the existence in $G$ of a bounded closed absolutely convex set $Q$ such that $Q$ is not absorbed by $V$. Take $y_{1} \in Q \sim V$. Suppose we have already constructed the elements $y_{1}, y_{2}, \ldots, y_{q}$ of $Q$ such that

$$
y_{p} \notin p V, y_{p}=\sum_{n \in N(p)} \lambda_{n} x_{n}, \lambda_{n} \in K, \quad p=1,2, \ldots, q,
$$

where

$$
\begin{gathered}
N(1)=1,2, \ldots, n_{1} \\
N(2)=n_{1}+1, n_{1}+2, \ldots, n_{2} \\
\ldots \ldots \ldots \ldots \ldots \ldots, \ldots \ldots \ldots, \ldots \\
N(q)=n_{q-1}+1, n_{q-1}+2, \ldots, n_{q} .
\end{gathered}
$$

If $G_{q}$ denotes the linear hull of

$$
\left\{x_{n}: n \in N, n \notin N(p), p=1,2, \ldots, q\right\}
$$

then $G_{q} \cap G_{Q}$ is a closed finite codimensional subspace of $G_{Q}$. If $X$ is a topological complement of $G_{q} \cap G_{Q}$ in $G_{Q}$, call $Q_{1}$ and $Q_{2}$ the projections of $Q$ over $X$ and $G_{q} \cap G_{Q}$, respectively. Obviously, $V$ absorbs $Q_{1}$ which is a bounded set in a finite dimensional space. Since $Q_{2}$ is a bounded set in $G_{q} \cap G_{Q}$ there is a $\lambda>0$ such that

$$
Q_{2} \subset \lambda\left(Q \cap G_{q}\right) \text {. }
$$

$V$ cannot absorb $Q \cap G_{q}$ because otherwise there is a $\mu>0$ such that

$$
Q_{1} \subset \mu V, Q \cap G_{q} \subset \mu V
$$

and therefore

$$
Q \subset Q_{1}+Q_{2} \subset Q_{1}+\lambda\left(Q \cap G_{q}\right) \subset \mu V+\lambda \mu V=\mu(1+\lambda) V,
$$

which is a contradiction. Thus, there is an element $y_{q+1}$ in $Q \cap G_{q}$ so that

$$
y_{q+1} \notin(q+1) V
$$


which can be written

$$
y_{q+1}=\sum_{n \in N(q+1)} \lambda_{n} x_{n}, \lambda_{n} \in K
$$

where

$$
N(q+1)=\left\{n_{q}+1, n_{q}+2, \ldots, n_{q+1}\right\} .
$$

The elements of the sequence $(N(q))$ are a partition of $N$. We write

$$
\begin{gathered}
M_{1}=\cup\{N(2 q-1): q=1,2, \ldots\} \\
M_{2}=\cup\{N(2 q): q=1,2, \ldots\} .
\end{gathered}
$$

Obviously, $M_{1} \cap M_{2}=\varnothing$ and $M_{1} \cup M_{2}=N$ and since $U$ is an ultrafilter it follows that, for instance, $M_{1}$ belongs to $U$. The sequence ( $\left.y_{2 q}\right)$ belongs to $L\left(M_{1}\right)$ and it is bounded. Writing $D$ for the closed absolutely convex hull of $\left(y_{2 q}\right)$ in $E$ we have that $E_{D} \cap L\left(M_{1}\right)$ is a Banach space since $E_{D}$ is a Banach space and therefore $V \cap E_{\left.D \cap L M_{1}\right)}$ is a neighbourhood of the origin in $E_{D \cap L\left(M_{1}\right)}$ (remember that $V$ is a $\mathscr{F}_{\text {-neighbourhood of the }}$ origin in $L$ ) and thus $V$ absorbs the elements of the sequence $\left(y_{2 q}\right)$ which is in contradiction with $y_{2 q} \notin(2 q) V$.

Proposition 3. If $G$ is a bormological space then $L$ is an ultrabornological space.

Proof. Let $J$ be the canonical injection of $G$ into $L[\mathscr{F}]$. The former proposition implies that $J$ is continuous since if $V$ is an absolutely convex $\mathscr{T}$-neighbourhood of the origin in $L$, then $J^{-1}(V)=V \cap G$ absorbs the bounded sets of $G$ and since $G$ is bornological $J^{-1}(V)$ is a neighbourhood of the origin in $G$. If $L[\mathscr{F}]$ is the completion of $L[\mathscr{T}], J$ can be extended to a continuous linear mapping $\varphi$ from $L$ into $L[\mathscr{T}]$. The conclusion follows if we show that $\varphi$ is the identity from $L$ in $L[\mathscr{T}]$. But this is the case since if $z \in L$ there is $U \in \mathcal{U}$ such that $z \in L(U)$. According to Proposition 1, $L(U) \cap G$ is $\mathscr{F}$-dense in $L(U)$ and therefore there is a net

$$
\left\{z_{i}: i \in I, \geqslant\right\}
$$

in $L(U) \cap G \mathscr{F}$-converging to $z$ which obviously also converges to $z$ in $L$ and thus

$$
\lim \left\{\varphi\left(z_{i}\right): i \in L, \geqslant\right\}=\varphi(z)=\lim \left\{J\left(z_{i}\right): i \in I, \geqslant\right\}=\lim \left\{z_{i}: i \in I, \geqslant\right\}=z .
$$

Note 3. In (2) ultrafilters on $N$ are used also to construct examples of metrizable ultrabarrelled spaces which are not Baire-hyperplane spaces.

\section{Proposition 4. L is an hyperplane of $F$.}

Proof. Given any $x$ belongs to $L$ there is an element $U \in \mathcal{U}$ such that $x \in L(U)$ and therefore $\Delta(x) \notin \mathscr{U}$. On the other hand $\Delta\left(x_{0}\right) \in \mathscr{U}$ and thus $x_{0} \notin L$. Since $F$ is the linear hull of $L \cup\left\{x_{0}\right\}, L$ is an hyperplane of $F$.

Proposition 5. F is not an inductive limit of Baire-hyperplane spaces. 
Proof. Suppose that there is a family $\left\{\left(F_{i}, \varphi_{i}\right): i \in I\right\}$ such that $F_{i}$ is a Bairehyperplane space, $\varphi_{i}$ is a linear continuous mapping from $F_{i}$ into $F$ and $F$ is the locally convex hull of the family. Let $u$ be a linear form on $F$ such that $u^{-1}(0)=L$. Since $u$ is not continuous there is an index $h \in I$ such that $\mu{ }^{\circ} \varphi_{h}$ is not continuous on $F_{h}$ and thus $\varphi_{h}^{-1}(L)$ is a dense hyperplane of $F_{h}$. Take a net $\left\{y_{j} ; j \in J, \geqslant\right\}$ in $\varphi_{h}^{-1}(L)$ converging in $F_{h}$ to an element $y_{0} \varphi_{h}^{-1}(L)$ and select a sequence $\left(t_{n}\right)$ such that if $\varphi_{h}\left(y_{0}\right)=z_{0}$ and $\varphi_{h}\left(t_{n}\right)=z_{n}$ then

$$
\lim _{n \rightarrow \infty} u_{m}\left(z_{n}\right)=u_{m}\left(z_{0}\right), m=1,2, \ldots
$$

Write $z_{0}=\lambda x_{0}+x, \lambda \neq 0, x \in L$ and then

$$
\Delta\left(z_{0}\right) \supset \Delta\left(\lambda x_{0}\right) \sim \Delta(x)=\Delta\left(x_{0}\right) \sim \Delta(x)
$$

and since $\Delta\left(x_{0}\right) \in \mathcal{U}$ and $\Delta(x) \notin \mathcal{U}$ it follows that $\Delta\left(z_{0}\right) \in \mathscr{U}$. Write

$$
\begin{gathered}
Q_{1}=\Delta\left(z_{1}\right) \cap \Delta\left(z_{0}\right) \\
Q_{m}=\left[\Delta\left(z_{m}\right) \sim \bigcup_{p=1}^{m-1} \Delta\left(z_{p}\right)\right] \cap \Delta\left(z_{0}\right), \quad m=2,3, \ldots
\end{gathered}
$$

If $n \in \Delta\left(z_{0}\right)$ the sequence $\left(u_{n}\left(z_{p}\right)\right)_{p=1}^{\infty}$ converges to $u_{n}\left(z_{0}\right)$ and therefore there is a positive integer $p_{0}$ such that

$$
u_{n}\left(z_{p}\right) \neq 0 \text {, for } p \geqslant p_{0} \text {. }
$$

If $u_{n}\left(z_{r}\right)$ is the first element of $\left(u_{n}\left(z_{p}\right)\right)_{p=1}^{\infty}$ which is non-zero then $n \in Q_{r}$ and therefore

$$
\Delta\left(z_{0}\right)=\cup\left\{Q_{p}: p=1,2, \ldots\right\} \text {. }
$$

Since

$$
Q_{p} \subset \Delta\left(z_{p}\right) \notin \mathcal{U},
$$

given a finite subset $M$ of $\mathrm{N}$ the union

$$
\cup\left\{Q_{p}: p \in M\right\} \notin \mathcal{U}
$$

and thus

$$
\cup\left\{Q_{p}: p \in M\right\} \neq \Delta\left(z_{0}\right)
$$

and therefore there is a strictly increasing sequence of positive integers $(n(q))_{q=1}^{\infty}$ such that

$$
Q_{n(q)} \neq \emptyset, q=1,2, \ldots
$$

hence we can suppose that the sequence $\left(z_{n}\right)$ has been selected so that $Q_{n} \neq \emptyset$, $n=1,2, \ldots$.

For any pair of positive integers $n, m$ construct a continuous linear form $\psi_{n m}$ on $F_{h}$ such that

$$
\psi_{n m}(t)=u_{n}\left(z_{0}\right) u_{m}\left(\varphi_{h}(t)\right)-u_{n}\left(\varphi_{h}(t)\right) u_{m}\left(z_{0}\right), t \in F_{h} .
$$

Let $\left\{f_{n}: n \in P \subset N\right\}$ be the set of all the non-null elements of $\left\{\psi_{n m}: n, m \in N\right\}$. If $p, q$ are positive integers, $p<q$, and if $n \in Q_{p}, m \in Q_{q}$, then $m \notin \Delta\left(z_{p}\right)$ and $n \in \Delta\left(z_{p}\right)$ and 
therefore $u_{m}\left(z_{p}\right)=0$ and $u_{n}\left(z_{p}\right) \neq 0$. Then

$$
\begin{aligned}
\psi_{n m}\left(t_{p}\right) & =u_{n}\left(z_{0}\right) u_{m}\left(\varphi_{h}\left(t_{p}\right)\right)-u_{n}\left(\varphi_{h}\left(t_{p}\right)\right) u_{m}\left(z_{0}\right)=u_{n}\left(z_{0}\right) u_{m}\left(z_{p}\right)-u_{n}\left(z_{p}\right) u_{m}\left(z_{0}\right) \\
& =-u_{n}\left(z_{p}\right) u_{m}\left(z_{0}\right) \neq 0,
\end{aligned}
$$

hence $\left\{f_{n}: n \in P \subset N\right\} \neq \emptyset$. Since $F_{h}$ is a Baire-hyperplane space the family $\left\{f_{n}^{-1}(0): n \in\right.$ $P$ \} of closed hyperplanes of $F_{h}$ does not cover $F_{h}$ and therefore there is $v \in F_{h}$ with $f_{n}(v) \neq 0$ for every $n \in P$.

The set $\Delta\left(\varphi_{h}(v)\right)$ belongs to $U$. Indeed, suppose that $\Delta\left(z_{0}\right) \ell \Delta\left(\varphi_{h}(v)\right)$, which is the difficult case. Then there is $q \in \Delta\left(z_{0}\right)$ so that $u_{q}\left(\varphi_{h}(v)\right)=0$. Take a positive integer $k$ such that $q \in Q_{k}$. If $p \in N, p \neq k$, let $m$ be an element of $Q_{p}$. Then, according to (1), we can take $s \in P$ such that $\psi_{q m}=f_{s}$ and therefore

$$
0 \neq f_{s}(v)=\psi_{q m}(v)=u_{q}\left(z_{0}\right) u_{m}\left(\varphi_{h}(v)\right)-u_{q}\left(\varphi_{h}(v)\right) u_{m}\left(z_{0}\right)=u_{q}\left(z_{0}\right) u_{m}\left(\varphi_{h}(v)\right)
$$

and therefore $u_{m}\left(\varphi_{h}(v)\right) \neq 0$ and thus

$$
\Delta\left(\varphi_{h}(v)\right) \supset \Delta\left(z_{0}\right) \sim Q_{k},
$$

hence $\left(\varphi_{h}(v)\right) \in \mathcal{U}$.

Write

$$
\varphi_{h}(v)=\rho z_{0}+w, \quad \rho \neq 0, w \in L .
$$

Since $\Delta(\omega) \notin \mathscr{U}$ there are two different $p, q \in N$ such that

$$
\left(\Delta\left(z_{0}\right) \sim \Delta(w)\right) \cap Q_{p} \neq \not,\left(\Delta\left(z_{0}\right) \sim \Delta(z)\right) \cap Q_{q} \neq \not \varnothing .
$$

Take $n \in Q_{p}$ and $m \in Q_{q}$. Then

$$
\begin{gathered}
u_{n}\left(\varphi_{h}(v)\right)=\rho u_{n}\left(z_{0}\right)+u_{n}(w)=\rho u_{n}\left(z_{0}\right) \\
u_{m}\left(\varphi_{h}(v)\right)=\rho u_{m}\left(z_{0}\right)+u_{m}(w)=\rho u_{m}\left(z_{0}\right)
\end{gathered}
$$

and therefore

$$
0 \neq \psi_{n m}(v)=u_{n}\left(z_{0}\right) u_{m}\left(\varphi_{h}(v)\right)-u_{n}\left(\varphi_{h}(v)\right) u_{m}\left(z_{0}\right)=u_{n}\left(z_{0}\right) \rho u_{m}\left(z_{0}\right)-\rho u_{n}\left(z_{0}\right) u_{m}\left(z_{0}\right)=0,
$$

which is a contradiction.

Proposition 6. If a barrelled space $H$ admits a bi-orthogonal sequence $\left(x_{n}, u_{n}\right)$ such that the linear hull $M$ of $\left(x_{n}\right)$ is not closed in $H$ then there is an element $x \in H$ so that the sequence $\left(u_{n}(x)\right)$ has an infinity of non vanishing terms.

Proof. If $Z$ denotes the subspace of $H$ orthogonal to $\left\{u_{1}, u_{2}, \ldots, u_{n}, \ldots\right\}$ the sequence $\left(u_{n}(z)\right)$, for any $z \in H$, has a finite number of non vanishing terms if and only if $z \in M+Z$. Suppose that $H$ coincides with $M+Z$. If $\varphi$ denotes the canonical mapping from $H$ onto $H / Z$ and $\psi$ stands for the restriction of $\varphi$ to $M$ then $\psi$ is an injective continuous mapping from $M$ onto $H / Z$. Since $H / Z$ is a barrelled space of countable dimension it is provided with the finest locally convex topology and therefore also $M$ which implies its completeness but $M$ was supposed to be non closed in $E$. 
Note 4. If $E$ is a space with a dense subspace $G$ of infinite countable dimension, a theorem of Klee $(4$, p. 148$)$ can be applied to obtain a bi-orthogonal system $\left(x_{n}, u_{n}\right)$ for $E$ so that the linear hull of $\left(x_{n}\right)$ is $G$. If $E$ is locally complete and $G$ is bornological and different from $E$ there is an element $x_{0} \in E$ such that $\Delta\left(x_{0}\right)$ is infinite, according to Proposition 6, and the spaces $L$ and $F$ can be constructed with the properties obtained in Propositions 3, 4, 5.

Theorem 6. Let $E$ be a separable Fréchet space of infinite dimension. Then there are two dense subspaces $L$ and $F$ of $E$ so that

1. $L$ is an hyperplane of $F$.

2. $L$ is ultrabornological.

3. Fis a barrelled space which is not an inductive limit of Baire-hyperplane space.

Proof. Since $E$ is a separable Fréchet space of infinite dimension there is an infinite countable dimensional dense subspace which is different from $E$. According to Note 4 the theorem follows.

Theorem 7. Let $E$ be the inductive limit of a sequence of infinite dimensional separable Fréchet spaces. If $E$ is locally complete there are two dense subspaces $L$ and $F$ of $E$ so that

1. $L$ is an hyperplane of $F$.

2. $L$ is ultrabornological.

3. F is a bornological barrelled space which is not an inductive limit of Bairehyperplane spaces.

Proof. Let $\left(E_{n}\right)$ be an increasing sequence of subspaces of $E$ with union $E$. Let $\mathscr{F}_{n}$ be a topology on $E_{n}$, finer than the original topology, such that $E_{n}\left[\mathscr{F}_{n}\right]$ is an infinite dimensional separable Fréchet space. Take in $E_{n}\left[\mathscr{F}_{n}\right]$ a dense subspace $G_{n}$ of countable dimension. Then the linear hull $G$ of $\cup\left\{G_{n}: n=1,2, \ldots\right\}$ is dense in $E$ and different from $E$. On the other hand, it is easy to check that $G$ is the inductive limit of the sequence of metrizable spaces $\left(G_{n}\right)$ and thus $G$ is bornological. We apply Note 4 and we finish by showing that $F$ is bornological. Indeed, if we suppose that $F \cap E_{n}$ is provided with the topology induced by $\mathscr{F}_{n}$ it is easy to show that $F$ is the inductive limit of the sequence of metrizable spaces $\left(F \cap E_{n}\right)$.

Proposition 7. $I \cdot H$ be a subspace of $\mathscr{D}^{\prime}(\Omega)$ such that it contains $\mathscr{D}_{0}^{\prime}(\Omega)$. Then $H$ is bornological.

Proof. If $B$ is a bounded set of $H$ there is a compact absolutely convex set $A$ in $\mathscr{D}^{\prime}(\Omega)$ such that $B$ is a precompact set of $\mathscr{D}^{\prime}(\Omega)_{A}$ since $\mathscr{D}^{\prime}(\Omega)$ is a nuclear complete space. Then $B$ is a precompact set of $\mathscr{D}^{\prime}(\Omega)_{A \cap H}$ and therefore there is in $\mathscr{D}^{\prime}(\Omega)_{A \cap H}$ a sequence converging to the origin with closed absolutely convex hull containing $B$ (3, p. 273). Then the topology $\mathscr{F}$ on $\mathscr{D}(\Omega)$ of the uniform convergence of the sequences of $H$ converging to the origin in the sense of Mackey coincides with the topology on $\mathscr{D}(\Omega)$ of the uniform convergence of the bounded sets of $H$.

Let $\left\{\varphi_{i}: i \in I, \geqslant\right\}$ be a Cauchy net in $\mathscr{D}(\Omega)$ for the topology $\mathscr{F}$. Let $M$ be the set of 
all the points of $\Omega$ with rational coordinates. If $b \in M, T_{m}(b)$ is the set of the delta of Dirac $\delta_{b}$ concentrated in $b$ and all its derivatives $\delta_{b}^{(m)}$ with order $|m|$ less or equal than n. Let

$$
T_{n}=\cup\left\{T_{n}(b): b \in M\right\} .
$$

Then $T_{n}$ is a bounded set of $H$ and thus given $\epsilon>0$ and $p=\left(p_{1}, p_{2}, \ldots, p_{m}\right)$ where $p_{r}$ is a non negative integer, $r=1,2, \ldots, m$, there is an $i_{0} \in I$ such that

$$
\left|\left\langle\varphi_{i}-\varphi_{i}, \delta b^{\left.()^{\prime}\right)}\right\rangle\right|=\left|\varphi_{i}^{\left.()^{\prime}\right)}(b)-\varphi_{i}^{(p)}(b)\right| \leqslant \epsilon, i, j \geqslant i_{0}, \forall b \in M
$$

and since $\varphi_{i}, i \in I$, is a function on $\Omega$ with values in $K$ having continuous derivatives of all orders we have that

$$
\left|\varphi_{i}^{(p)}(x)-\varphi^{(\rho)}(x)\right| \leqslant \epsilon, \forall x \in \Omega,
$$

and therefore there is a function $\varphi$ on $\Omega$ with values in $K$ having continuous derivatives of all orders such that

$$
\lim \left\{\varphi_{i}^{(p)}(x): i \in T, \geqslant\right\}=\varphi^{(p)}(x)
$$

uniformly on $\Omega$. Suppose that $\varphi$ has non compact support in $\Omega$. Then there is a sequence $\left(x_{n}\right)$ in $\Omega$ not contained in any compact of $\Omega$ such that $\varphi\left(x_{n}\right) \neq 0, n=$ $1,2, \ldots$ The subset of $H$

$$
\left\{\frac{1}{\varphi\left(x_{n}\right)} \delta_{x_{n}}: n=1,2, \ldots\right\}
$$

is bounded and therefore given a positive $\epsilon$ less than 1 there is an index $k \in I$ such that

$$
\left|\left\langle\varphi_{k}-\varphi, \frac{1}{\varphi\left(x_{n}\right)} \delta_{x_{n}}\right\rangle\right|=\left|\frac{1}{\varphi\left(x_{n}\right)} \varphi_{k}\left(x_{n}\right)-1\right|<\epsilon, n=1,2, \ldots
$$

Since the support $D$ of $\varphi_{k}$ is compact there is a positive integer $q$ such that $x_{q} \notin D$ and thus

$$
\left|\frac{1}{\varphi\left(x_{q}\right)} \varphi_{k}\left(x_{q}\right)-1\right|=1<\epsilon<1
$$

which is a contradiction and therefore $\varphi \in \mathscr{D}(\Omega)$ and thus $\mathscr{D}(\Omega)[\mathscr{F}]$ is complete. Since $H$ is a Mackey space, (5), and according to a theorem of Köthe, $(3$, p. 386), $H$ is bornological.

Theorem 8. There are two dense subspaces $L$ and $F$ in $\mathscr{D}^{\prime}[\Omega]$ such that

1. $L$ is a hyperplane of $F$.

2. $L$ is ultrabornological.

3. $F$ is a bornological barrelled space which is not an inductive limit of Bairehyperplane spaces.

Proof. $\mathscr{D}_{0}^{\prime}(\Omega)$ has countable dimension, is dense in $\mathscr{D}(\Omega)$ and $\mathscr{D}_{0}^{\prime}(\Omega) \neq \mathscr{D}(\Omega)$. On the other hand, $\mathscr{D}(\Omega)$ is complete. Then, according to Note 4 , the theorem follows. 


\section{REFERENCES}

(1) I. AMEMIYA and Y. KoMURA, Über nicht-vollständige Montel-räume, Math. Ann. 177 (1968), 273-271.

(2) P. Dierolf, S. Dierolf and L. Drenowski, Remarks and examples concerning unordered Baire-like and ultrabarrelled spaces, (To be published).

(3) G. KöTHE, Topological Vector Spaces I, (Springer, Berlin-Heidelberg-New York, 1969).

(4) J. T. MARTI, Introduction to the theory of bases, (Springer, Berlin, 1969).

(5) M. VAldiviA, On Mackey spaces, Duke Math. J. 41 (1974) 835-841.

\section{Facultad de Ciencias}

PASEO Al Mar. 13

VALENCIA

SPAIN 